\title{
Research on correlation of compositions with oestrogenic activity of Cistanche based on LC/Q- TOF-MS/MS technology
}

https://doi.org/10.1515/chem-2019-0001

received March 20, 2018; accepted July 28, 2018.

\begin{abstract}
LC technology is a recognized method used worldwide to evaluate the quality of traditional Chinese medicines (TCM). The quality of TCM has a direct impact on its efficacy. Therefore, in order to thoroughly reveal how TCM exerts its efficacy, first of all, it is necessary to understand the material basis for its efficacy, and then to control the quality of active compounds. The application of the spectrum-effect relationship method is crucial for determining the pharmacological material basis. The goal of this paper was to investigate the underlying correlations between the chemical profiles and oestrogenic activity of Cistanche, to reveal the active compounds. The chemical profiles of Cistanche were recorded using HPLC/QTOF-MS/MS, and oestrogenic activity was determined by the Uterus growth test and the MTT assay. Then combining the results of bivariate analysis, principal component analysis and gray correlation analysis method, fifteen active compounds were identified. They are 8-epiloganic acid, salidroside, syringalide A 3'- $\alpha$-l-rhamnopyranoside, cistanoside A, echinacoside, cistanoside $\mathrm{F}$, cistanoside $\mathrm{B}$, cistanoside $\mathrm{C}$, osmanthuside $\mathrm{B}$, acteoside, isoacteoside, tubuloside B, 2'-acetylacteoside, and two unknown compounds. This study lays a foundation for in vivo studies of Cistanche, and for the development of its clinical application.
\end{abstract}

\footnotetext{
*Corresponding author: Wen-Lan Li, School of Pharmacy, Harbin University of Commerce, Harbin 150076, China, E-mail: liwenlan2010@163.com

Jing-Xin Ding, Jing Bai, Hui Song, Yu-Bin Ji: Research Center on Life Sciences and Environmental Sciences, Harbin University of Commerce, Harbin 150076, China; Engineering Research Center of Natural Anticancer Drugs, Ministry of Education, Harbin 150076, China

Yang Hu, Xiang-Ming Sun: School of Pharmacy, Harbin University of Commerce, Harbin 150076, China
}

Keywords: Cistanche deserticola; LC/Q-TOF-MS; Oestrogenic activity; principal component analysis (PCA); Uterus growth test.

\section{Abbreviations}

$\begin{array}{ll}\text { TCM } & \text { traditional Chinese medicines } \\ \text { HPLC/Q-TOF-MS } & \begin{array}{l}\text { High-performance liquid } \\ \text { chromatography/quadrupole time-of- } \\ \text { flight mass spectrometry } \\ \text { bivariate analysis } \\ \text { principal component analysis } \\ \text { BA }\end{array} \\ \text { PCA } & \text { gray correlation analysis method } \\ \text { GCAM } & \text { Trypsin, 3-(4, 5-Dimethylthiazol-2-yl)-2, } \\ \text { MTT } & \text { 5-diphenyltetrazoliumbromide } \\ \text { DMSO } & \text { dimethyl sulfoxide } \\ \text { RPMI1640 } & \text { Roswell Park Memorial Institute 1640 }\end{array}$

\section{Introduction}

Recently, TCM has become more and more popular in Asian and Western countries due to their stable therapeutic effects and low toxicity in the clinic. As a result, it is necessary to develop novel types of TCM and to understand the effective compositions used during their clinical application [1-6].

Firstly, the spectrum-effect relationship is studied to discern the correlation between chromatographic fingerprint and pharmacodynamics efficacy. Then using the relationship to look for effective components in TCM, and to formulate control standards to reflect their internal quality. The spectrum-effect relationship has been applied in many areas of TCM research, such as material basis of single and compound TCM, component compatibility, processing mechanism, pharmacological effect forecast and technology optimization [7-12]. The spectrum-effect relationship is used to put forward new ideas and methods 
for exploring active substances, optimizing formulas, improving preparation processes, tracking and separating target ingredients, and for the development of new TCM drugs [13-17].

Cistanche has been widely used for treatment of sexual dysfunction, constipation, prostatitis and kidney deficiency. It has a wide spectrum of bioactivities including oestrogenic effect, anti-aging, antioxidation and immune action [18-19]. Generally, phenylethanoid glycosides (such as cistanoside A, tubuloside A), lignans, and iridoid glycosides were considered as pharmacological constituents and chemical markers for quality control of Cistanche and its products [20-21]. The chemical spectra of Cistanche from different areas were recorded using Highperformance liquid chromatography (HPLC) [22-23]. In this study, we select uterus growth test and the MCF7 cell proliferation assay to assess their oestrogenic activities. Principal component analysis, bivariate analysis and gray correlation analysis method were used to correlate oestrogenic activity with the individual components. The aim of this study was to identify bioactive compounds of Cistanche responsible for in vivo oestrogenic effects. The study lays a foundation for in-depth studies of Cistanche.

\section{Experimental Procedure}

\subsection{Materials}

Agilent 1290 HPLC system, Agilent 6530 Series Quadrupole Time-of-Flight LC/MS (Q-TOF) system and Chemical HPLC-3D workstations were used for analytical chemistry experiments and data processing. Milli- $Q$ ultrapure water was used for preparation of samples and standards. Additional instruments included the electronic Analytical Balance AR1140 (Ohaus International Ltd), the 680 Microplate Reader (Bio-Rad Corporation) and the 64R High-Speed Centrifuge (Beckman Coulter Allegra).

Immature female Kunming mice (about $21 \mathrm{~d}$ of birth, weaned) weighing $(12 \pm 2) \mathrm{g}$, were purchased from Changchun National Biological Industry Base Laboratory Animal Center (Changchun, China). The mice were housed in a temperature-regulated room $\left(22 \pm 2^{\circ} \mathrm{C}\right)$ with unrestricted access to food and water. Animal experimentation was initiated after five days of acclimatization. The mice were fasted overnight with free access to water before administering the test solutions. This study was carried out in strict accordance with the recommendations in the Guide for the Care and Use of Laboratory Animals of the National Institutes of Health.
All experimental procedures were reviewed and approved by the Animal Ethical Committee of Harbin University of Commerce.

Cistanche deserticola (cd-20120227-005) was purchased from drug market, and identified by Prof. Zhang Delian (Harbin University of Commerce). Diethylstilbestrol was purchased from HEFEI JIULIAN PHARMACEUTICAL CO.,LTD. RPMI1640 and RPMI1640 without phenol red were purchased from HyClone Company (USA). MTT and DMSO were all purchased from Sigma-Aldrich Co. LLC (St Louis, USA). The human breast cancer MCF7 cell line was provided by the Research Center of the Life Sciences and Environmental Sciences (Harbin University of Commerce). The standards used were as follows: acteoside (111530200505), echinacoside (111670-200503) and salidroside (110736-200628) were obtained from the National Institute for the Control of Pharmaceutical and Biological Products (Beijing, China). Acetonitrile, methanol and formic acid were of MS-grade, whereas water was ultrapure. Other reagents were of analytical grade and were commercially available.

\subsection{Preparation of the sample solution}

Cistanche samples were collected from ten diverse habitats, namely Neimeng, Xinjiang, Ningxia, Guizhou, Chaidamu, Xizang, Alashan, Qinghai, Sichuan and Hubei Province.

The solution $(1.0 \mathrm{~g} / \mathrm{mL})$ of the Cistanche sample was prepared in distilled water for oral administration. The concentration of diethylstilboestrol was $20 \mu \mathrm{g} / \mathrm{mL}$, and was used as the positive control solution. The solution $(1.0 \mathrm{~g} / \mathrm{mL})$ of Cistanche sample was prepared in 50\% acetonitrile. Acteoside, echinacoside and salidroside (2.0 $\mathrm{mg}$ each) were dissolved in $10 \mathrm{~mL}$ of $50 \%$ acetonitrile solution. Both the sample and standard solutions were filtered using a $0.45-\mu \mathrm{m}$ filter prior to analysis.

\subsection{LC-MS conditions}

A Waters Symmetry Shield RP18 $(3.9 \mathrm{~mm} \times 150 \mathrm{~mm}, 5 \mu \mathrm{m}$; Waters Corporation, Milford, MA, USA) was used to analyse the samples. The mobile phase consisted of acetonitrile (A) and $0.2 \%(v / v)$ formic acid aqueous solution (B), and was pumped at a flow rate of $0.5 \mathrm{~mL} / \mathrm{min}$. The injection volume of each sample was $10 \mu \mathrm{L}$. The gradient elution program was as follows: Mobile phase A was initiated at $5 \%$ and increased linearly to $23 \%$ at $35 \mathrm{~min}$. Solution A was subsequently increased to $25 \%$ ( 35 to $65 \mathrm{~min}$ ). The 
mobile phase A was then decreased to $5 \%$ for the last 5 min of the run ( 65 to $70 \mathrm{~min}$ ). The column temperature was maintained at $30^{\circ} \mathrm{C}$. The chromatograms were monitored at $254 \mathrm{~nm}$. The atomization gas pressure was $30 \mathrm{psi}$, and the capillary voltage was $3.5 \mathrm{kV}$. The flow rate of the dry gas was set to $8 \mathrm{~L} / \mathrm{min}$ with the temperature at $30^{\circ} \mathrm{C}$. The mass spectra were recorded within the scan range of 100$3,000 \mathrm{Da}$, and were in negative ion scanning mode.

\subsection{Fingerprint evaluation}

LC fingerprints of the samples collected from 10 different batches were established, and matched automatically using the Similarity Evaluation System for Chromatographic Fingerprint of TCM (version 2012; China Pharmacopoeia Committee, Beijing, China). Furthermore, the reference chromatogram map was generated using the median method. In addition, cluster analysis was applied to evaluate the quality of the samples from ten diverse habitats.

\subsection{Principal component analysis (PCA)}

PCA is a multivariate statistical method. It can retain sufficient information from the data acquisition. In the present study, characteristic peaks from LC chromatograms were screened further by PCA and aimed to identify active compounds based on spectrum-effect relationship.

\subsection{Uterus growth test}

The mice were divided into 12 groups (10 per group). The sample solutions that were prepared from Cistanche were collected from ten diverse habitats and were administered twice daily (morning and evening) by gavage at a dose of $30 \mathrm{~g} / \mathrm{kg}$ body weight for four days. The blank and positive control groups comprised equal volumes of distilled water and diethylstilboestrol solution and were administered twice daily for four days. On the fifth day, the drugcontaining blood samples were withdrawn from the anaesthetized mice. The samples were centrifuged (5,000 $\mathrm{rpm}, 10 \mathrm{~min}$ ), and the supernatants (drug-containing serum samples) were separated. The drug-containing serum samples were inactivated by heating at $56^{\circ} \mathrm{C}$ in a water bath for $30 \mathrm{~min}$, and were filtered through a $0.22-\mu \mathrm{m}$ filter prior to the MTT assay. Concomitantly, the uteri of the mice were immediately excised and weighed in order to calculate the uterine weight indices. The animal experimentation was initiated following five days of acclimatization. The mice were fasted overnight with free access to water prior to administration of the test solutions. The present study was carried out in strict accordance with the recommendations of the Guide for the Care and Use of Laboratory Animals of the National Institutes of Health. All experimental procedures were reviewed and approved by the Animal Ethical Committee of the Harbin University of Commerce. All potentially stressful procedures were performed under sodium pentobarbital anaesthesia.

\subsection{MTT assay}

MCF7 cells were co-cultured with RPMI 1640 without phenol red (containing 5\% CDT-FBS) for four days. The cells were seeded in 96-well plates at a density of 2,000 cells/well. An adherent culture was achieved and following $72 \mathrm{~h}$ of culture, the cells were co-cultured in RPMI 1640 medium containing serum samples from Cistanche-treated of mice. The experiments were conducted in six replicates. Subsequently, $20 \mu \mathrm{L}$ of MTT solution $(5 \mathrm{mg} / \mathrm{mL}$ in PBS) was added in each well, and the cells were incubated for $4 \mathrm{~h}$. Following medium removal, $150 \mu \mathrm{L}$ of DMSO was added in order to produce the formazan product, and the plates were shaken in the dark to completely dissolve. The absorbance was measured at $570 \mathrm{~nm}$ using a Microplate Reader. The proliferation rate was calculated as the average absorbance value of the drug-containing serum group divided by the average absorbance value of the blank control group (cells co-cultured in RPMI 1640 without serum).

\subsection{Statistical analysis}

The paired-sample t-test (two-tailed) was used to identify statistically significant differences among values in the blank control and experimental groups. The differences were considered significant at 95\% confidence level ( $\mathrm{p}<$ 0.05).

PCA was used to evaluate characteristic peak areas in the chromatograms of the samples from ten diverse habitats. Bivariate analysis and Gray correlation analysis were used to assess the correlation of peak areas and their oestrogenic activity. The correlation was calculated by SPSS statistics software statistics (SPSS for Windows 21.0, SPSS Inc., USA). The differences were considered significant at 95\% confidence level (two-tailed).

Ethical approval: The conducted research is not related to either human or animal use. 


\section{Results and discussion}

\subsection{Cluster analysis and similarity assessment}

The relative standard deviation of the retention time and peak area of characteristic peaks were 0.58 and $0.34 \%$ for precision, 0.52 and $0.31 \%$ for reproducibility, and 0.19 and $0.13 \%$ for stability, respectively. These findingsindicated that our LC method of fingerprint establishment was reasonable and reliable. The similarities of the chromatograms of ten samples were greater than 0.9. The common peaks for all samples could not fully represent characteristic constituents of Cistanche, since the peak could possibly have been lost. Therefore, the sample matching number was adjusted from 26 to 20 in order to obtain a more accurate chromatogram map. A total of 26 peaks were obtained, and the sum of their areas was greater than 0.8 compared with those of the overall peak area in the sample. Therefore, we concluded that these chromatographic peaks could effectively reflect main chemical constituents of Cistanche. The results of cluster analysis are shown in Figure 1. A total of ten diverse habitats were divided into three categories as follows: (A) 2, Guizhou; 3, Qinghai; 4,Chaidamu; 10, Neimeng; 7, Xinjiang and 6, Sichuan. (B) 1, Xizang; 8, Ningxia and 5, Alashan. (C) 9, Hubei. The trend of cluster analysis showed that the habitats of the medicinal herbs is similar when at the bottom of the threshold. But with the increasing of the threshold, the climate and growth conditions of habitats are close. It shows that the cluster analysis can not only distinguish the diverse habitats well, but also reflect the affinities between them.

\subsection{Identification of chemical constituents}

Based on the synergy between components, TCM exerted pharmacological effects. Certain studies have been conducted to identify the chemical compositions of $C$. deserticola [24-26]. The correlation of inherent constituents with their activities indicated that they could be identified based on the combination of LC with the corresponding indices of pharmacological activity. Q-TOF-MS is suitable for multi-constituent identification of TCM, since it can provide the exact molecular mass because of its high resolution. A total of 26 compounds were identified by analyzing the primary and secondary MS data. PCA was used to assess whether these compounds with regard to their oestrogenic activity. A contribution of the activity is indicated by an eigen value higher than $1(>1)$ and a

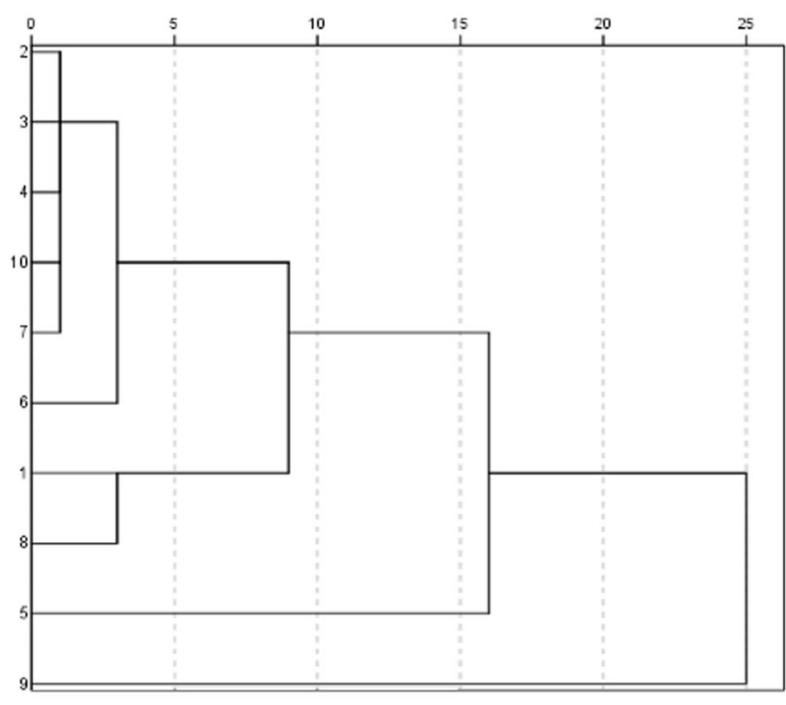

Figure 1: Cluster analysis of Cistanche from ten diverse habitats, which were grouped into three clusters: (A) 2, Guizhou; 3, Qinghai; 4,Chaidamu; 10, Neimeng; 7, Xinjiang; and 6, Sichuan. (B) 1, Xizang; 8, Ningxia; and 5, Alashan. (C) 9, Hubei.

cumulative contribution rate of variables higher than 0.8 ( $>0.8$ ). The higher, the absolute value of the eigen load, the higher the influence of the compounds on the oestrogenic indices.

The chromatograms of Cistanche and the standard samples were compared in order to identify individual components under the same LC-MS conditions. As shown in Table 1, a total of 26 constituents were identified based on the retention time and MS data [24-26]. Active ingredients are oral administrated in the form of mixtures rather than monomers as traditional Chinese medicine is in the form of a mixture to play a therapeutic role. A single compound after being individually separated was not found to play a role. Our goal is to reveal compounds that have oestrogenic activity in the known components of Cistanche [23-30], and therefore did not select NMR and other technical means to validate their identity. Peak 1 indicated a predominant deprotonated ion with a $m / z$ ratio of $373\left(\mathrm{C}_{16} \mathrm{H}_{22} \mathrm{O}_{10}\right)$, which was identical to the elemental composition of geniposidic acid. This was supported by the loss of a glucose moiety that yielded a fragment ion with a $\mathrm{m} / \mathrm{z}$ ratio of 211 . Peak 2 indicated a predominant deprotonated ion with a $\mathrm{m} / z$ ratio of $461\left(\mathrm{C}_{20} \mathrm{H}_{30} \mathrm{O}_{12}\right)$, which was identical to the elemental composition of decaffeoylacteoside, and was supported by the loss of a rhamnosyl moiety, forming a fragment ion with a $\mathrm{m} / z$ ratio of 315 . Peak 4 indicated a predominant deprotonated ion with a $m / z$ ratio of $375\left(\mathrm{C}_{16} \mathrm{H}_{24} \mathrm{O}_{10}\right)$, which was identical to the elemental composition of 8-epiloganic acid. The loss of a glucose moiety, formed a fragment ion with a $m / z$ ratio of 213 , further supported theinference. Peak 
Table 1: Identification of chemical composition of the peaks.

\begin{tabular}{|c|c|c|c|c|c|}
\hline Peak & $t_{R}(\min )^{a}$ & $\begin{array}{l}\text { negative } \mathrm{m} \\
{[\mathrm{M}-\mathrm{H}]^{-}(\mathrm{m} / \mathrm{z}}\end{array}$ & $\begin{array}{l}\text { lode }(m / z) \\
z)^{\text {b }} \text { MS }^{2}(m / z)\end{array}$ & Formula & Compound presumed \\
\hline 1 & 18.502 & 373.1135 & $\begin{array}{l}211.0607 \\
123.0448\end{array}$ & $\mathrm{C}_{16} \mathrm{H}_{22} \mathrm{O}_{10}$ & geniposidic acid \\
\hline 2 & 19.981 & 461.1675 & 315.1085 & $\mathrm{C}_{20} \mathrm{H}_{30} \mathrm{O}_{12}$ & decaffeoylacteoside \\
\hline 3 & 22.714 & 633.2066 & 487.1466 & - & unknown \\
\hline 4 & 23.476 & 375.1305 & 213.0783 & $\mathrm{C}_{16} \mathrm{H}_{24} \mathrm{O}_{10}$ & 8-epiloganic acid \\
\hline 5 & 24.641 & 299.3084 & 219.8404 & $\mathrm{C}_{14} \mathrm{H}_{20} \mathrm{O}_{7}$ & salidroside \\
\hline 6 & 26.299 & 607.2248 & 445.0720 & $\mathrm{C}_{29} \mathrm{H}_{36} \mathrm{O}_{14}$ & syringalide A 3'- $\alpha$ - l-rhamnopyranoside \\
\hline 7 & 27.240 & 435.1515 & 227.0915 & - & unknown \\
\hline 8 & 28.673 & 799.2626 & 637.2139 & $\mathrm{C}_{36} \mathrm{H}_{48} \mathrm{O}_{20}$ & cistanoside A \\
\hline 9 & 29.390 & 811.2679 & $\begin{array}{l}665.2097 \\
503.1771\end{array}$ & $\mathrm{C}_{37} \mathrm{H}_{48} \mathrm{O}_{20}$ & Kankanoside $\mathrm{H}_{1}$ \\
\hline 10 & 32.303 & 785.2623 & 623.2037 & $\mathrm{C}_{35} \mathrm{H}_{46} \mathrm{O}_{20}$ & echinacoside \\
\hline 11 & 34.229 & 487.1466 & $\begin{array}{l}341.1091 \\
179.0340\end{array}$ & $\mathrm{C}_{21} \mathrm{H}_{28} \mathrm{O}_{13}$ & cistanoside $\mathrm{F}$ \\
\hline 12 & 34.812 & 813.2844 & 651.1939 & $\mathrm{C}_{37} \mathrm{H}_{50} \mathrm{O}_{20}$ & cistanoside B \\
\hline 13 & 36.246 & 827.2629 & 681.2035 & $\mathrm{C}_{37} \mathrm{H}_{48} \mathrm{O}_{21}$ & tubuloside $\mathrm{A}$ \\
\hline 14 & 37.142 & 637.2164 & 491.2733 & $\mathrm{C}_{30} \mathrm{H}_{38} \mathrm{O}_{15}$ & cistanoside C \\
\hline 15 & 37.769 & 591.2091 & $\begin{array}{l}445.0762 \\
145.0290\end{array}$ & $\mathrm{C}_{29} \mathrm{H}_{36} \mathrm{O}_{13}$ & osmanthuside B \\
\hline 16 & 39.293 & 623.2255 & 461.1615 & $\mathrm{C}_{29} \mathrm{H}_{36} \mathrm{O}_{15}$ & acteoside \\
\hline 17 & 39.517 & 623.2108 & 461.1632 & $\mathrm{C}_{29} \mathrm{H}_{36} \mathrm{O}_{15}$ & cis-acteoside \\
\hline 18 & 40.995 & 623.1991 & 461.1675 & $\mathrm{C}_{29} \mathrm{H}_{36} \mathrm{O}_{15}$ & isoacteoside \\
\hline 19 & 41.667 & 769.2642 & $\begin{array}{l}623.2046 \\
487.1453\end{array}$ & $\mathrm{C}_{35} \mathrm{H}_{46} \mathrm{O}_{19}$ & cistantubuloside B \\
\hline 20 & 43.370 & 651.2293 & 459.1324 & $\mathrm{C}_{31} \mathrm{H}_{40} \mathrm{O}_{15}$ & cistanoside D \\
\hline 21 & 45.969 & 653.2293 & $\begin{array}{l}491.2713 \\
345.1006\end{array}$ & $\mathrm{C}_{30} \mathrm{H}_{38} \mathrm{O}_{16}$ & campneoside 圆 \\
\hline 22 & 46.910 & 665.2014 & $\begin{array}{l}623.1659 \\
461.1226\end{array}$ & $\mathrm{C}_{31} \mathrm{H}_{38} \mathrm{O}_{16}$ & tubuloside B \\
\hline 23 & 47.224 & 639.1942 & 493.2337 & $\mathrm{C}_{29} \mathrm{H}_{36} \mathrm{O}_{16}$ & campneoside 目 \\
\hline 24 & 49.419 & 665.2153 & $\begin{array}{l}623.1981 \\
461.1662\end{array}$ & $\mathrm{C}_{31} \mathrm{H}_{38} \mathrm{O}_{16}$ & 2'-acetylacteoside \\
\hline 25 & 50.987 & 445.0785 & $\begin{array}{l}299.3017 \\
268.0447\end{array}$ & $\mathrm{C}_{20} \mathrm{H}_{30} \mathrm{O}_{11}$ & cistanoside G \\
\hline 26 & 52.556 & 347.1720 & 179.0551 & $\mathrm{C}_{16} \mathrm{H}_{28} \mathrm{O}_{8}$ & kankanoside E \\
\hline
\end{tabular}

${ }^{a}$ retention time

${ }^{\mathrm{b}}$ quasi-molecular ion

${ }^{\mathrm{c}} \mathrm{MS}$ fragment ions 
6 indicated a predominant deprotonated ion with a $\mathrm{m} / \mathrm{z}$ ratio of $607\left(\mathrm{C}_{29} \mathrm{H}_{36} \mathrm{O}_{14}\right)$, which was identical to the elemental composition of syringalide $A 3$ ' $\alpha$ - 1-rhamnopyranoside. The loss of a caff moiety, formed a fragment ion with a $\mathrm{m} / \mathrm{z}$ ratio of 445 , further supported the inference. Peak 8 indicated a predominant deprotonated ion with a $\mathrm{m} / z$ ratio of $799\left(\mathrm{C}_{36} \mathrm{H}_{48} \mathrm{O}_{20}\right)$, which was identical to the elemental composition of cistanoside A. The loss of a caff moiety, formed a fragment ion with a $\mathrm{m} / \mathrm{z}$ ratio of 637 , further supported the inference. Peak 11 indicated a predominant deprotonated ion with a $\mathrm{m} / z$ ratio of $487\left(\mathrm{C}_{21} \mathrm{H}_{28} \mathrm{O}_{13}\right)$, which was identical to the elemental composition of cistanoside F. This was supported by the loss of a rhamnosyl moiety that yielded a fragment ion with a $\mathrm{m} / \mathrm{z}$ ratio of 341 , and the loss of another caff moiety formed a fragment ion with a $\mathrm{m} / \mathrm{z}$ ratio of 179 , further supported the inference. Peak 12 indicated a predominant deprotonated ion with a $\mathrm{m} / z$ ratio of $813\left(\mathrm{C}_{37} \mathrm{H}_{50} \mathrm{O}_{20}\right)$, which was identical to the elemental composition of cistanoside B. The loss of a glucose moiety, formed a fragment ion with a $\mathrm{m} / \mathrm{z}$ ratio of 651 , further supported the inference. Peak 13 indicated a predominant deprotonated ion with a $\mathrm{m} / z$ ratio of $827\left(\mathrm{C}_{37} \mathrm{H}_{48} \mathrm{O}_{21}\right)$, which was identical to the elemental composition of tubuloside A. The loss of a rhamnosyl moiety, formed a fragment ion with a $m / z$ ratio of 681 , further supported the inference. Peak 14 indicated a predominant deprotonated ion with a $\mathrm{m} / z$ ratio of $637\left(\mathrm{C}_{30} \mathrm{H}_{38} \mathrm{O}_{15}\right)$, which was identical to the elemental composition of cistanoside $\mathrm{C}$. The loss of a rhamnosyl moiety, formed a fragment ion with a $\mathrm{m} / \mathrm{z}$ ratio of 491, further supported the inference. Peak 15 indicated a predominant deprotonated ion with a $\mathrm{m} / \mathrm{z}$ ratio of $591\left(\mathrm{C}_{29} \mathrm{H}_{36} \mathrm{O}_{13}\right)$, which was identical to the elemental composition of osmanthuside B. The loss of a rhamnosyl moiety, formed a fragment ion with a $m / z$ ratio of 445 , further supported the inference. Peak 16 indicated a predominant deprotonated ion with a $m / z$ ratio of $623\left(\mathrm{C}_{29} \mathrm{H}_{36} \mathrm{O}_{15}\right)$, which was identical to the elemental composition of acteoside. The loss of a caff moiety, formed a fragment ion with a $\mathrm{m} / \mathrm{z}$ ratio of 461 , further supported the inference. Peak 17 indicated a predominant deprotonated ion with a $\mathrm{m} / z$ ratio of $623\left(\mathrm{C}_{29} \mathrm{H}_{36} \mathrm{O}_{15}\right)$, which was identical to the elemental composition of cis-acteoside. The loss of a caff moiety, formed a fragment ion with a $\mathrm{m} / \mathrm{z}$ ratio of 461 , further supported the inference. Peak 18 indicated a predominant deprotonated ion with a $m / z$ ratio of $623\left(\mathrm{C}_{29} \mathrm{H}_{36} \mathrm{O}_{15}\right)$, which was identical to the elemental composition of isoacteoside. The loss of a caff moiety, formed a fragment ion with a $\mathrm{m} / \mathrm{z}$ ratio of 461 , further supported the inference. Peak 21 indicated a predominant deprotonated ion with a $\mathrm{m} / z$ ratio of $653\left(\mathrm{C}_{30} \mathrm{H}_{38} \mathrm{O}_{16}\right)$, which was identical to the elemental composition of campneoside . The loss of a caff moiety, formed a fragment ion with a $\mathrm{m} / \mathrm{z}$ ratio of 491, and the loss of another rhamnosyl moiety formed a fragment ion with a $m / z$ ratio of 345 , further supported the inference. Peak 22 indicated a predominant deprotonated ion with a $m / z$ ratio of $665\left(\mathrm{C}_{31} \mathrm{H}_{38} \mathrm{O}_{16}\right)$, which was identical to the elemental composition of tubuloside $\mathrm{B}$. The loss of a $\mathrm{CH}_{3} \mathrm{CO}$ moiety, formed a fragment ion with a $\mathrm{m} / \mathrm{z}$ ratio of 623, and the loss of another caff moiety formed a fragment ion with a $m / z$ ratio of 461 , further supported the inference. Peak 23 indicated a predominant deprotonated ion with a $m / z$ ratio of $639\left(\mathrm{C}_{29} \mathrm{H}_{36} \mathrm{O}_{16}\right)$, which was identical to the elemental composition of campneoside . The loss of a rhamnosyl moiety, formed a fragment ion with a $m / z$ ratio of 493 , further supported the inference. Peak 24 indicated a predominant deprotonated ion with a $\mathrm{m} / z$ ratio of $665\left(\mathrm{C}_{31} \mathrm{H}_{38} \mathrm{O}_{16}\right)$, which was identical to the elemental composition of 2'-acetylacteoside. The loss of an Ac moiety, formed a fragment ion with a $m / z$ ratio of 623 , and the loss of another caff moiety formed a fragment ion with a $\mathrm{m} / \mathrm{z}$ ratio of 461 , further supported the inference. Peak 25 indicated a predominant deprotonated ion with a $\mathrm{m} / z$ ratio of $445\left(\mathrm{C}_{20} \mathrm{H}_{30} \mathrm{O}_{11}\right)$, which was identical to the elemental composition of cistanoside $\mathrm{G}$. The loss of a rhamnosyl moiety, formed a fragment ion with a $m / z$ ratio of 299, further supported the inference. The structures of compounds are listed in Figure 2.

\subsection{PCA}

As shown in Table 2, the eigen values of the first eight principal components were higher than $1(>1)$, and the cumulative contribution rate of the first six principal components was $93.417 \%$. Therefore, the first six principal components were evaluated further. Table 2 indicates their eigen values and factor loadings. The first principal component exhibited higher load based on the eigen values of the eight peaks $(>0.9)$. These peaks were the following: $9,12,14$, and 15. Consequently, echinacoside, cistanoside $\mathrm{B}$, osmanthuside B and acteoside, corresponded to a total of 4 peaks were selected as representative variables for oestrogenic activity (Figure 3).

\subsection{Determination of oestrogenic activity}

The ratio of uterine wet weight to body weight of each mouse was calculated (Figure 4). The animals exposed to Cistanche from nine habitats exhibited significant oestrogenic activities by increasing their uterine weight compared with mice in the blank control group. Figure 4 


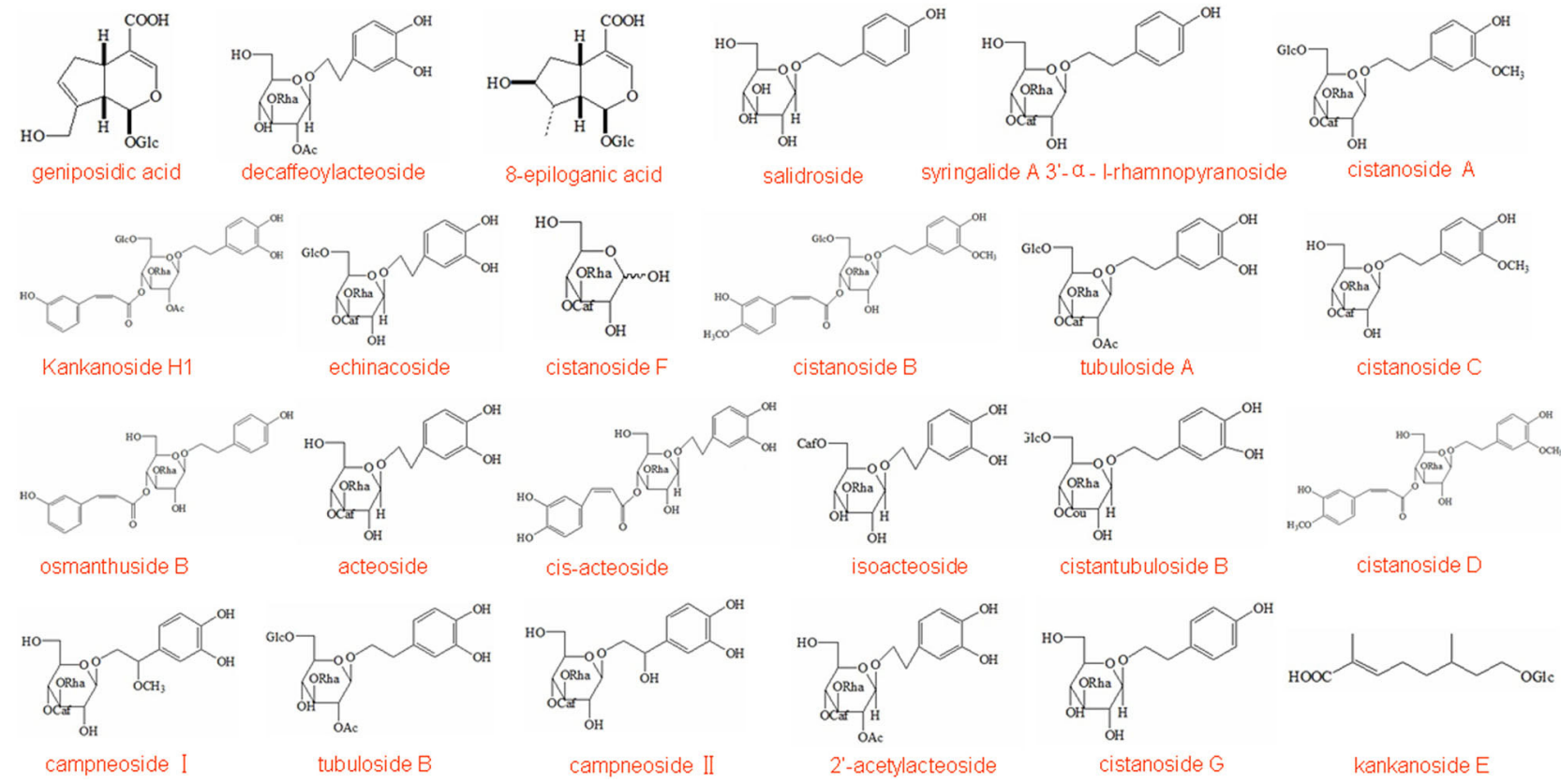

Figure 2: The structure of compounds.

Table 2: Eigen values and corresponding percentages of the variables (top nine principal components)

\begin{tabular}{lllllll}
\hline Component & \multicolumn{2}{l}{ Initial eigen values } & & \multicolumn{3}{l}{ Extraction sums of squared loadings } \\
& Total & Variance(\%) & Cumulative (\%) & Total & Variance (\%) & Cumulative (\%) \\
\hline 1 & 9.085 & 43.261 & 43.261 & 9.085 & 43.261 & 43.261 \\
2 & 3.374 & 16.068 & 59.329 & 3.374 & 16.068 & 59.329 \\
3 & 2.729 & 12.997 & 72.326 & 2.729 & 12.997 & 72.326 \\
4 & 2.056 & 9.793 & 82.118 & 2.056 & 9.793 & 82.118 \\
5 & 1.311 & 6.243 & 88.361 & 1.311 & 6.243 & 88.361 \\
6 & 1.062 & 5.056 & 93.417 & 1.062 & 5.056 & 93.417 \\
7 & 0.675 & 3.215 & 96.632 & & & \\
8 & 0.501 & 2.385 & 99.017 & & & \\
\hline
\end{tabular}

further shows the proliferation rate of MCF7 cells treated with the serum of Cistanche-treated mice. The cells treated with the sera of mice that were administered Cistanche from ten habitats exhibited significantly higher growth compared with cells devoid of treatment.

\subsection{Bivariate analysis}

In the present study, we observed that the uterine weights of immature female Kunming mice increased and the proliferation of MCF7 human breast cancer cells was promoted. These findings verified the oestrogenic activity of Cistanche. Figure 5 indicated the uterine indices and MCF7 cell proliferation rates of these constituents. Pearson's correlation coefficient was used to calculate the relative peak areas, which corresponded to oestrogenic activity of the samples. The correlation coefficients higher than 0.3 were considered significant $(p<0.05)$, whereas correlation coefficients higher than 0.5 were considered highly significantly $(\mathrm{p}<0.01)$. Based on the results, Cistanche was shown to possess oestrogenic activity. 


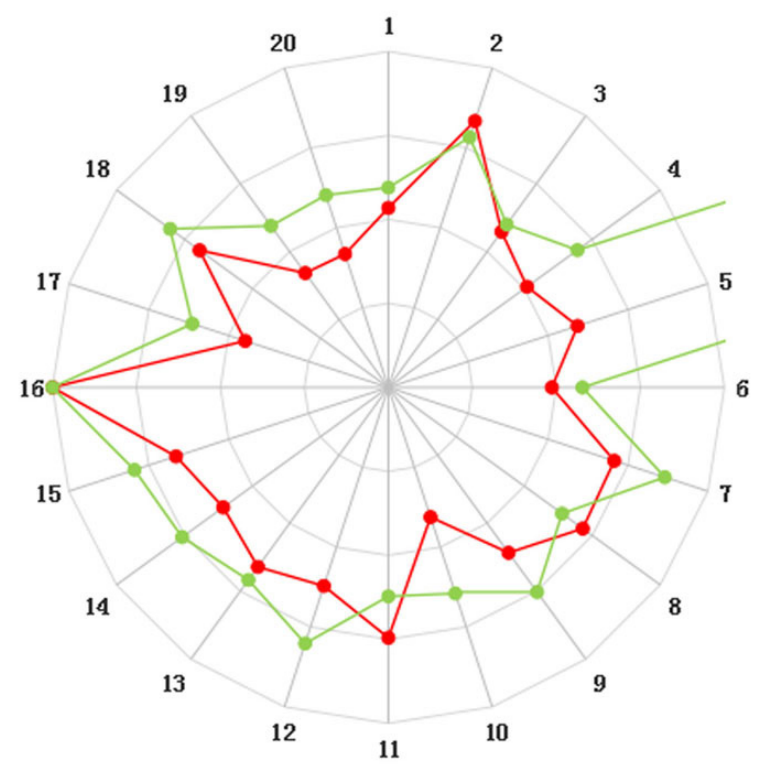

Figure 3: The scatter plots of the compounds identified in Cistanche based on principal component analysis (PCA). The red line is the uterine indices, and the green line is the MCF7 cell proliferation. The first principal component exhibited higher load based on the eigen values of the eight peaks (>0.9). These peaks were the following: $9,12,14$, and 15 . Consequently, echinacoside, cistanoside B, osmanthuside B and acteoside, corresponded to a total of 4 peaks were selected as representative variables for oestrogenic activity.
A total of nine compounds significantly correlated with the increase in the uterine weight $(\mathrm{p}<0.05)$, whereas ten compounds significantly correlated with MCF7 cellular proliferation $(p<0.05)$. Compounds that significantly correlated with uterine weight and cell proliferation were 8-epiloganic acid, salidroside, syringalide A3'- $\alpha$ 1-rhamnopyranoside, cistanoside A, cistanoside B, cistanoside $\mathrm{C}$, osmanthuside $\mathrm{B}$, acteoside, isoacteoside, tubuloside B, 2'-acetylacteoside, and two unknown compounds (Figure 5). These thirteen compounds exerted oestrogenic activities.

\subsection{Gray correlation analysis method}

Figure 6 shows the relative correlation of the characteristic peaks of 26 compounds with the uterine indices, and Figure 7 shows the relative correlation of characteristic peaks of the 26 compounds with their cell proliferation rates. A total of three compounds exhibited the highest correlation with the uterine indices, namely 8-epiloganic acid, cistanoside F, and acteoside (Figure 6, in descending order); whereas the six compounds that exhibited the highest correlation with the MCF7 cell proliferation were echinacoside, cistanoside F, cistanoside B, osmanthuside

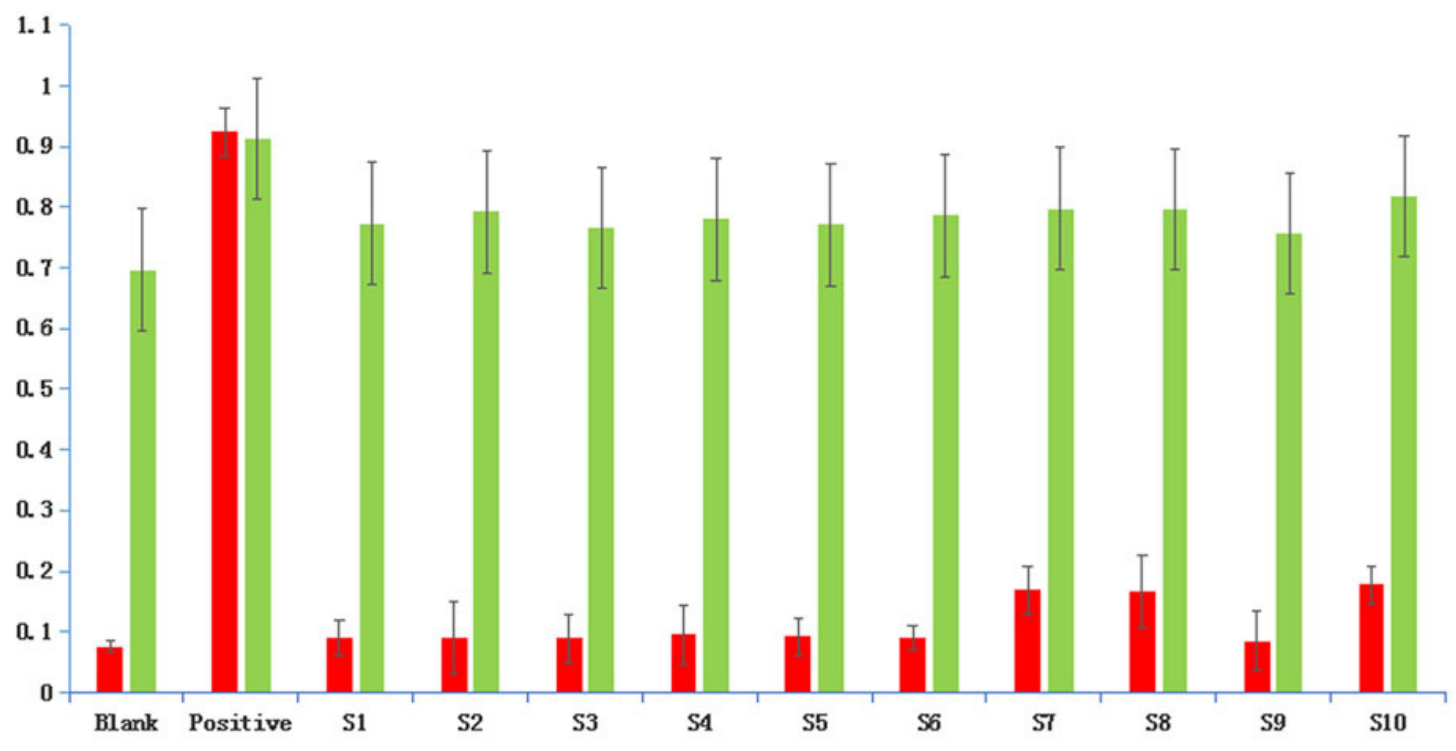

Figure 4: Percentage uterine wet weight relative to body weight in mice of different experimental groups $(n=10)$ and the proliferation of human breast cancer MCF7 cells following exposure to the serum of mice treated with Cistanche from ten different habits $(n=6)$. The red column is the uterine indices, and the green column is the MCF7 cell proliferation. S1, Xizang; S2, Guizhou; S3, Qinghai; S4, Chaidamu; S5, Alashan; S6, Sichuan; S7, Xinjiang; S8, Ningxia; S9, Hubei; and S10, Neimeng. The animals exposed to Cistanche from nine habitats exhibited significant oestrogenic activities by increasing their uterine weight compared with mice in the blank control group. The cells treated with the sera of mice that were administered Cistanche from ten habitats exhibited significantly higher growth compared with cells devoid of treatment. 


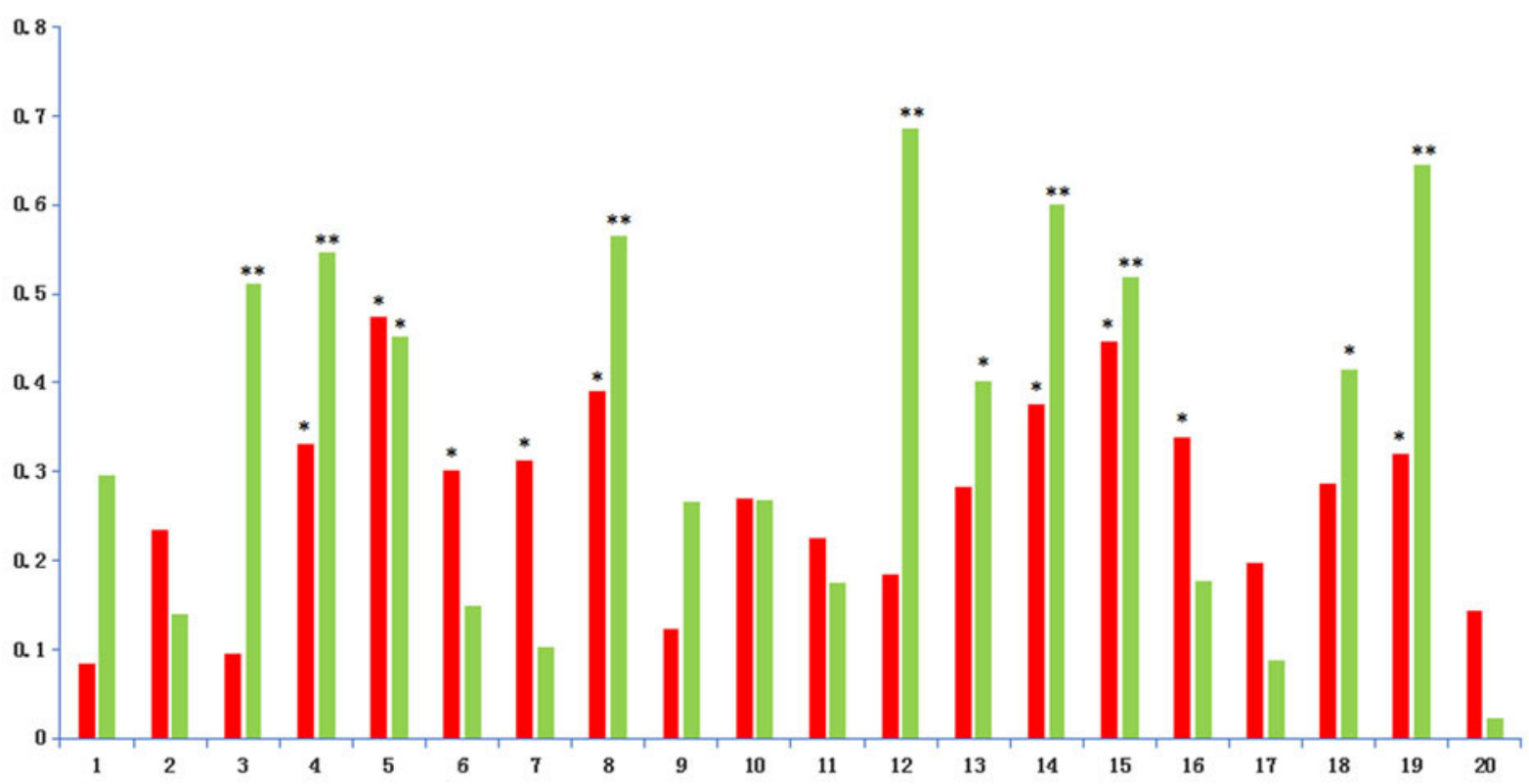

Figure 5: Bivariate analysis of the compounds identified in Cistanche. SPSS version 21.0 was used to analyze the data. The red column is the uterine indices, and the green column is the MCF7 cell proliferation. The correlation coefficients higher than 0.3 were considered significant $\left({ }^{*} p<0.05\right)$, whereas correlation coefficients higher than 0.5 were considered highly significantly $(* \star p<0.01)$. These thirteen compounds exerted oestrogenic activities. Compounds that significantly correlated with uterine weight and cell proliferation were 8-epiloganic acid, salidroside, syringalide A3'- $\alpha$ - I-rhamnopyranoside, cistanoside A, cistanoside B, cistanoside C, osmanthuside B, acteoside, isoacteoside, tubuloside B, 2'-acetylacteoside, and two unknown compounds.

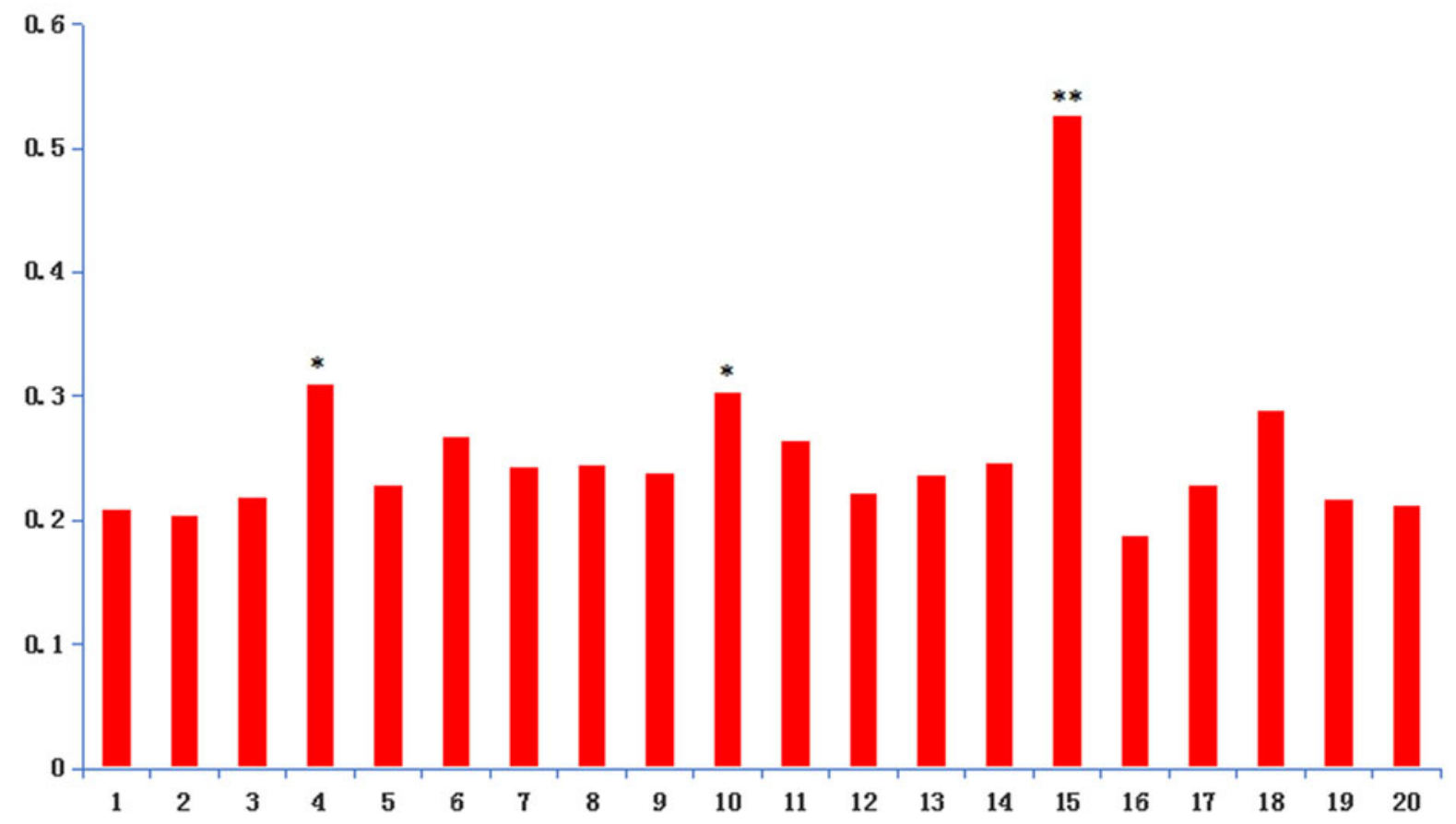

Figure 6: Gray correlation analysis method of the compounds identified in Cistanche. SPSS version 21.0 was used to analyze the data. The red column is the uterine indices. The correlation coefficients higher than 0.3 were considered significant $\left({ }^{\star} \mathrm{p}<0.05\right)$, whereas correlation coefficients higher than 0.5 were considered highly significantly $\left({ }^{\star *} \mathrm{p}<0.01\right)$. A total of three compounds exhibited the highest correlation with the uterine indices, namely 8 -epiloganic acid, cistanoside $\mathrm{F}$, and acteoside. 


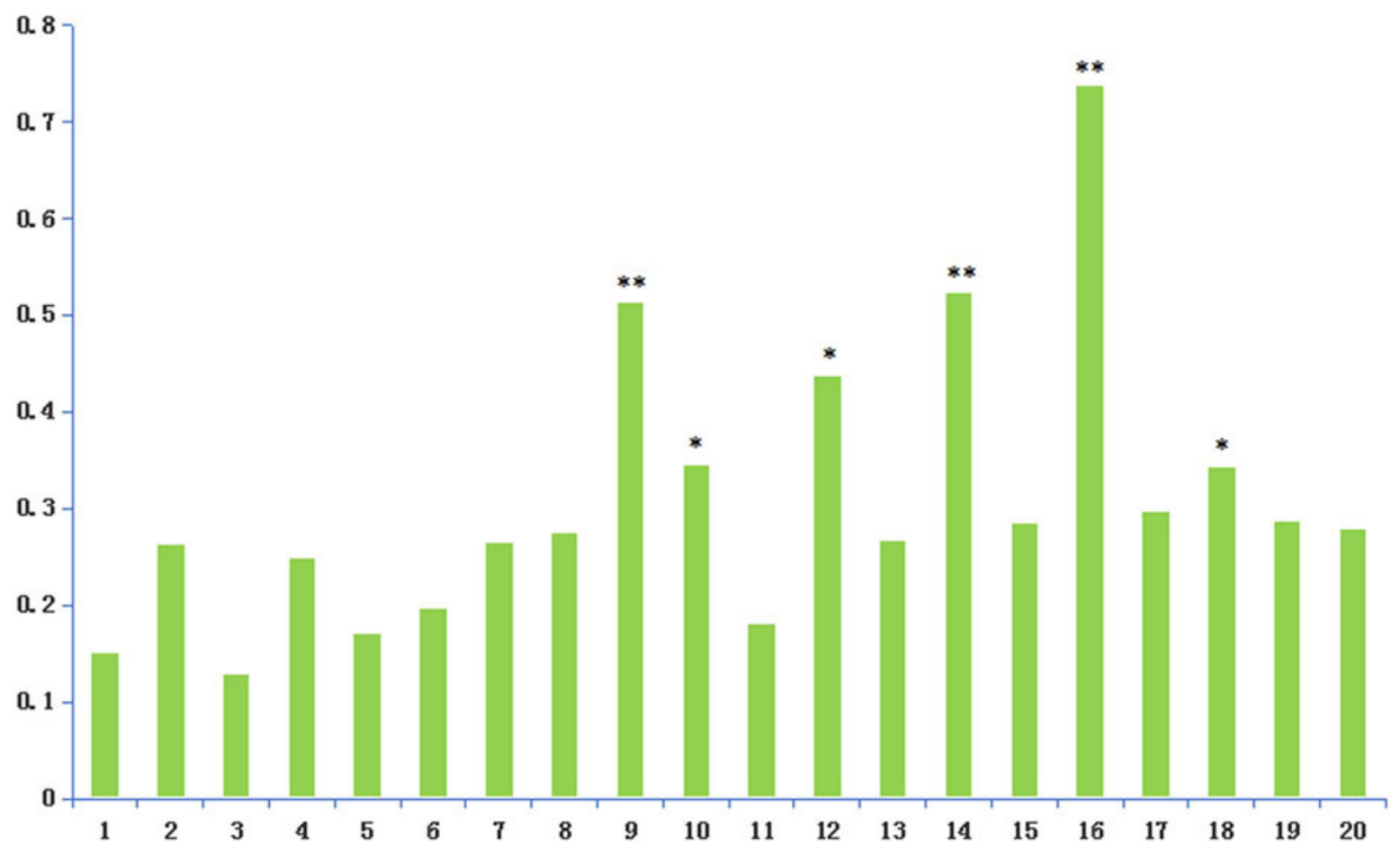

Figure 7: Gray correlation analysis method of the compounds identified in Cistanche. SPSS version 21.0 was used to analyze the data. The green column is the MCF7 cell proliferation. The correlation coefficients higher than 0.3 were considered significant $\left({ }^{*} p<0.05\right)$, whereas correlation coefficients higher than 0.5 were considered highly significantly ( ${ }^{*} \mathrm{p}<0.01$ ). A total of six compounds that exhibited the highest correlation with MCF7 cell proliferation were echinacoside, cistanoside F, cistanoside B, osmanthuside B, isoacteoside, and tubuloside B.

B, isoacteoside, and tubuloside B (Figure 7, in descending order). The relative correlations of echinacoside, osmanthuside $\mathrm{B}$, acteoside, and isoacteoside were higher than 0.5 .

\subsection{Optimization of LC-MS conditions}

To obtain a better chromatogram map, methanol and acetonitrile were compared. In final, the combination of aqueous formic acid $(0.2 \%, \mathrm{v} / \mathrm{v})$ - acetonitrile was the best mobile phase for separation. By in the range of 200-400nm full wavelength scanning, $254 \mathrm{~nm}$ was selected as the optimal. In addition, MS parameters were also improved. The negative ion scanning mode was selected, because it improved the response values of most ingredients in Cistanche.

\section{Conclusion}

LC-Q-TOF-MS technology was used to identify oestrogenic active constituents of Cistanche. The present study illustrated that 8-epiloganic acid, salidroside, syringalideA3'- $\alpha$-l-rhamnopyranoside, cistanoside A, echinacoside, cistanoside $\mathrm{F}$, cistanoside $\mathrm{B}$, cistanoside $\mathrm{C}$, osmanthuside $\mathrm{B}$, acteoside, isoacteoside, tubuloside $\mathrm{B}$, 2'-acetylacteoside, and two unknown compounds were the main active constituents of Cistanche. However, these findings require further studies in order explore the in vivo effects and mechanism of action of the constituents.

Acknowledgements: This project was supported by the National Natural Science Foundation of China (No. 81073015), Nature Scientific Foundation of Heilongjiang Province (ZD2017014), and the Young innovative talent training plan of the College in the Heilongjiang Province (UNPYSCT-2017209). The authors declare that there is no conflict of interest regarding the publication of this paper.

Conflict of interest: Authors state no conflict of interest. 


\section{References}

[1] Wang Z., Xia Q., Liu X., Liu W., Huang W., Mei X., Luo J., Shan M., Lin R., Zou D., Ma Z., Phytochemistry, pharmacology, quality control and future research of Forsythia suspensa (Thunb.) Vahl: A review, J. Ethnopharmacol., 2018, 210, 318339.

[2] Shu Y., Liu Z., Zhao S., Song Z., He D., Wang M., Zeng H., Lu C., Lu A., Liu Y., Integrated and global pseudotargeted metabolomics strategy applied to screening for quality control markers of Citrus TCMs, Anal. Bioanal. Chem., 2017, 409(20), 4849-4865.

[3] Yang B., Wang Y., Shan L., Zou J., Wu Y., Yang F., Zhang Y., Li Y., Zhang Y., A Novel and Practical Chromatographic "FingerprintROC-SVM" Strategy Applied to Quality Analysis of Traditional Chinese Medicine Injections: Using KuDieZi Injection as a Case Study, Molecules., 2017, 22(7). pii: E1237.

[4] Zhuo L., Peng J., Zhao Y., Li D., Xie X., Tong L., Yu Z., Screening bioactive quality control markers of QiShenYiQi dripping pills based on the relationship between the ultra-high performance liquid chromatography fingerprint and vascular protective activity, J. Sep. Sci., 2017, 40(20), 4076-4084.

[5] Liu N., Li J., Li B.G., Application of multivariate statistical analysis and thinking in quality control of Chinese medicine, Zhongguo. Zhong. Yao. Za. Zhi., 2014, 39(21), 4268-4271.

[6] Wang D.D., Liang J., Yang W.Z., Hou J.J., Yang M., Da J., Wang Y., Jiang B.H., Liu X., Wu W.Y., Guo D.A., HPLC/qTOF-MSoriented characteristic components data set and chemometric analysis for the holistic quality control of complex TCM preparations: Niuhuang Shangqing pill as an example, J. Pharm. Biomed. Anal., 2014, 89, 130-141.

[7] Hernaez R., Thrift A.P., High Negative Predictive Value, Low Prevalence, and Spectrum Effect: Caution in the Interpretation, Clin. Gastroenterol. Hepatol., 2017, 15(9), 1355-1358.

[8] Liang J., Chen Y., Ren G., Dong W., Shi M., Xiong L., Li J., Dong J., Li F., Yuan J., Screening Hepatotoxic Components in Euodia rutaecarpa by UHPLC-QTOF/MS Based on the Spectrum-Toxicity Relationship, Molecules., 2017, 22(8). pii: E1264.

[9] Li W., Sun X., Liu B., Zhang L., Fan Z., Ji Y., Screening and identification of hepatotoxic component in Evodia rutaecarpa based on spectrum-effect relationship and UPLC-Q-TOFMS, Biomed. Chromatogr., 2016, 30(12), 1975-1983.

[10] Chopard G., Puyraveau M., Binetruy M., Meyer A., Vandel P., Magnin E., Berger E., Galmiche J., Mauny F., Spectrum Effect and Spectrum Bias in the Screening Test Performance for Amnestic Mild Cognitive Impairment: What are the Clinical Implications?, J. Alzheimers. Dis., 2015, 48(2), 385-393.

[11] Xu G.L., Xie M., Yang X.Y., Song Y., Yan C., Yang Y., Zhang X., Liu Z.Z., Tian Y.X., Wang Y., Jiang R., Liu W.R., Wang X.H., She G.M., Spectrum-effect relationships as a systematic approach to traditional chinese medicine research: current status and future perspectives, Molecules., 2014, 19(11), 17897-17925.

[12] Zheng Q., Zhao Y., Wang J., Liu T., Zhang B., Gong M., Li J., Liu H., Han B., Zhang Y., Song X., Li Y., Xiao X., Spectrum-effect relationships between UPLC fingerprints and bioactivities of crude secondary roots of Aconitum carmichaelii Debeaux (Fuzi) and its three processed products on mitochondrial growth coupled with canonical correlation analysis, J. Ethnopharmacol., 2014,153(3), 615-623.
[13] Shi Z., Liu Z., Liu C., Wu M., Su H., Ma X., Zang Y., Wang J., Zhao Y., Xiao X., Spectrum-Effect Relationships Between Chemical Fingerprints and Antibacterial Effects of Lonicerae Japonicae Flos and Lonicerae Flos Base on UPLC and Microcalorimetry, Front. Pharmacol., 2016, 7, 12.

[14] Usher-Smith J.A., Sharp S.J., Griffin S.J., The spectrum effect in tests for risk prediction, screening, and diagnosis, B.M.J., 2016, 353, i3139.

[15] Chen Y., Yu H., Wu H., Pan Y., Wang K., Liu L., Jin Y., Zhang C., Tracing novel hemostatic compounds from heating products of total flavonoids in Flos Sophorae by spectrum-effect relationships and column chromatography, J. Sep. Sci., 2015, 38(10), 1691-1699.

[16] Liu X., Wang X.L., Wu L., Li H., Qin K.M., Cai H., Pei K., Liu T., Cai B.C., Investigation on the spectrum-effect relationships of Da-Huang-Fu-Zi-Tang in rats by UHPLC-ESI-Q-TOF-MS method, J. Ethnopharmacol., 2014, 154(3), 606-612.

[17] Xie R.F., Zhou X., Shi Z.N., Li Y.M., Li Z.C., Study on spectrumeffect relationship of rhizoma Rhei, cortex Magnoliae Officinalis, fructus Aurantii Immaturus and their formula, J. Chromatogr. Sci., 2013, 51(6), 524-532.

[18] Li F., Yang X., Yang Y., Guo C., Zhang C., Yang Z., Li P., Antiosteoporotic activity of echinacoside in ovariectomized rats, Phytomedicine., 2013, 20(6), 549-557.

[19] Xiong W.T., Gu L., Wang C., Sun H.X., Liu X., Anti-hyperglycemic and hypolipidemic effects of Cistanche tubulosa in type 2 diabetic db/db mice, J. Ethnopharmacol., 2013, 150(3), 935945.

[20] Morikawa T., Ninomiya K., Imamura M., Akaki J., Fujikura S., Pan Y., Yuan D., Yoshikawa M., Jia X., Li Z., Muraoka O., Acylated phenylethanoid glycosides, echinacoside and acteoside from Cistanche tubulosa, improve glucose tolerance in mice, J. Nat. Med., 2014, 68(3), 561-566.

[21] Nan Z.D., Zeng K.W., Shi S.P., Zhao M.B., Jiang Y., Tu P.F., Phenylethanoid glycosides with anti-inflammatory activities from the stems of Cistanche deserticola cultured in Tarim desert, Fitoterapia., 2013, 89, 167-174.

[22] Lu D., Zhang J., Yang Z., Liu H., Li S., Wu B., Ma Z., Quantitative analysis of Cistanches Herba using high-performance liquid chromatography coupled with diode array detection and highresolution mass spectrometry combined with chemometric methods, J. Sep. Sci., 2013, 36(12), 1945-1952.

[23] Han L., Boakye-Yiadom M., Liu E., Zhang Y., Li W., Song X., Fu F., Gao X., Structural characterisation and identification of phenylethanoid glycosides from Cistanches deserticola Y.C. Ma by UHPLC/ESI-QTOF-MS/MS, Phytochem. Anal., 2012, 23(6), 668-676.

[24] Xiang Y., Jing Z., Haixia W., Ruitao Y., Huaixiu W., Zenggen L., Lijuan M., Yiping W., Yanduo T., Antiproliferative Activity of Phenylpropanoids Isolated from Lagotis brevituba Maxim, Phytother. Res., 2017, 31(10), 1509-1520.

[25] Yan Y., Song Q., Chen X., Li J., Li P., Wang Y., Liu T., Song Y., Tu P., Simultaneous determination of components with wide polarity and content ranges in Cistanche tubulosa using serially coupled reverse phase-hydrophilic interaction chromatography-tandem mass spectrometry, J. Chromatogr. A., 2017, 1501, 39-50.

[26] Zou P., Song Y., Lei W., Li J., Tu P., Jiang Y., Application of 1H NMR-based metabolomics for discrimination of different parts 
and development of a new processing workflow for Cistanche deserticola, Acta. Pharm. Sin. B., 2017, 7(6), 647-656.

[27] Pan Y.N., Studies on the constituents and bioactivity of fresh Cistanche tubulosa, PhD thesis, Shenyang Pharmaceutical University, Shenyang, China, 2011.

[28] Cui Q., Pan Y., Xu X., Zhang W., Wu X., Qu S., Liu X., The metabolic profile of acteoside produced by human or rat intestinal bacteria or intestinal enzyme in vitro employed UPLCQ-TOF-MS, Fitoterapia., 2016, 109, 67-74.

[29] Cui Q., Pan Y., Bai X., Zhang W., Chen L., Liu X., Systematic characterization of the metabolites of echinacoside and acteoside from Cistanche tubulosa in rat plasma, bile, urine and feces based on UPLC-ESI-Q-TOF-MS, Biomed. Chromatogr., 2016(9), 1406-1415.

[30] Cui Q., Pan Y., Yan X., Qu B., Liu X., Xiao W., A metabolic way to investigate related hurdles causing poor bioavailability in oral delivery of isoacteoside in rats employing ultrahighperformance liquid chromatography/quadrupole time-of-flight tandem mass spectrometry, Rapid. Commun. Mass. Spectrom., 2017(4), 371-380. 